\title{
Avaliação da aplicação de técnicas geodésicas no monitoramento de estruturas civis
}

\author{
Evaluation of the application of geodetic techniques for monitoring civil structures
}

\author{
Ricardo Vilar Neves ${ }^{1}$ \\ Pedro Luis Faggion ${ }^{1}$ \\ Carlos Aurélio Nadal ${ }^{1}$ \\ Luis Augusto Koenig Veiga ${ }^{1}$
}

\begin{abstract}
${ }^{1}$ Departamento de Geomática, Universidade Federal do Paraná - UFPR, Curso de Pós-Graduação em Ciências Geodésicas - CPCG. Avenida Coronel Francisco Heráclito dos Santos, 210 Centro Politécnico - Jardim das Américas Caixa Postal 19001, 81.531-990 Curitiba, Paraná, Brasil.

ricvilar@yahoo.com.br, faggion@ufpr.br, cnadal@ufpr.br,kngveiga@ufpr.br
\end{abstract}

\begin{abstract}
RESUMO - O presente trabalho apresenta um estudo sobre monitoramento de estruturas com finalidade de determinar se existem deslocamentos de pontos localizados junto aos blocos que formam a barragem da Usina Hidroelétrica de Salto Caxias, localizada no município de Capitão Leônidas Marques, Estado do Paraná. Para o monitoramento foram instalados equipamentos sobre pilares que possuem sistema de centragem forçada engastados na rocha à jusante da barragem e determinadas às coordenadas dos alvos implantados junto a três físsuras (bloco 5, bloco 8 e bloco 11). Foram aplicados testes estatísticos para avaliar os resultados obtidos e para verificar se as variações das coordenadas são significativas. Pode-se concluir baseando-se nos testes aplicados e nos resultados obtidos que não houve deslocamentos significativos ao longo do tempo nos blocos que formam a barragem.
\end{abstract}

Palavras-chave - barragem, sistema de centragem forçada, levantamento geodésico, análise de movimentos.

ABSTRACT - The present work shows a study about the monitoring of structures with the finality of determining if there are movements of points on the blocks that form the Dam of Salto Caxias Power Plant, located nearby Capitão Leônidas Marques city, Paraná state. Were used for monitoring pillars that have forced centering system set: the rock below the dam for the installation of the equipment, it was determined the coordinates of the targets planted close to three cracks (block 5, block 8 and block 11) also it was done statistics tests to evaluate the results and to verify if the variations of the coordinates were significant. It can be conclude based on the applied tests and results obtained that there weren't significant movements along the time on the blocks that form the Dam.

Keywods - dam, forced centering system, geodesic survey, analysis of movements.

\section{INTRODUÇÃO}

Atualmente uma das tarefas mais importantes nas Usinas Hidroelétricas é o monitoramento de pontos para determinar mudanças em corpos deformáveis, seja em forma, dimensão ou posição. Estes deslocamentos são detectados, principalmente pelas variações de coordenadas dos pontos observados.

Monitorar uma estrutura, do ponto de vista do posicionamento geodésico, significa determinar e comparar as coordenadas de pontos em duas épocas distintas, e verificar se, dentro de certo nível de confiabilidade (significância), houveram variações nestas coordenadas (SILVEIRA, 2003).

Existem vários métodos para avaliar estes movimentos, sendo que os mais conhecidos são os métodos geodésicos e os estruturais.

No caso em estudo, uma rede de monitoramento foi implantada à jusante da barragem da Usina Hidroelétrica de Salto Caxias com a finalidade de avaliar a estrutura através de métodos geodésicos.
Considerando que a barragem de Salto Caxias é uma das maiores do Brasil construída utilizando Concreto Compactado a Rolo (CCR), iniciou-se um projeto de pesquisa e desenvolvimento financiado pela Agencia Nacional de Energia Elétrica (ANNEL) e pela Companhia Paranaense de Energia Elétrica (COPEL) objetivando determinar a estabilidade de pontos engastados nos blocos da barragem utilizando técnicas geodésicas.

$\mathrm{Na}$ bibliografia pesquisada sobre redes de monitoramento de estruturas, os procedimentos são divididos em quatro etapas (GRANEMANN, 2005).

A primeira, denominada especificação, requer um conhecimento a priori da grandeza dos movimentos, o qual é também, uma condição para estabelecer quais equipamentos devem ser utilizados.

A segunda etapa, denominada concepção, visa atingir a melhor configuração da rede de controle e dos pontos a serem monitorados.

A terceira etapa, denominada implementação, é a etapa de maior custo, pois envolve técnicas de medição e implantação de sistemas de centragem forçada para a instalação dos instrumentos. 
A quarta etapa, denominada análise, é a etapa na qual as observações são calculadas e estatisticamente analisadas visando determinar as reais variações das coordenadas.

\section{DESCRIÇÃO DA METODOLOGIA APLICADA NO MONITORAMENTO DAS FISSURAS}

$\mathrm{Na}$ estrutura da barragem da usina hidroelétrica de Salto Caxias, existem pontos de controle a serem monitorados, pontos estes definidos pela equipe técnica da Companhia Paranaense de Energia Elétrica - COPEL. Trata-se de fissuras da estrutura, à montante e jusante da barragem, que devem ser monitorados de modo a verificar se há ou não movimentação significativa.

A rede externa de monitoramento é utilizada para avaliar a estabilidade da região onde está localizada a barragem e para o monitoramento das fissuras à jusante a partir do pilar P3, conforme a Fig. 1.

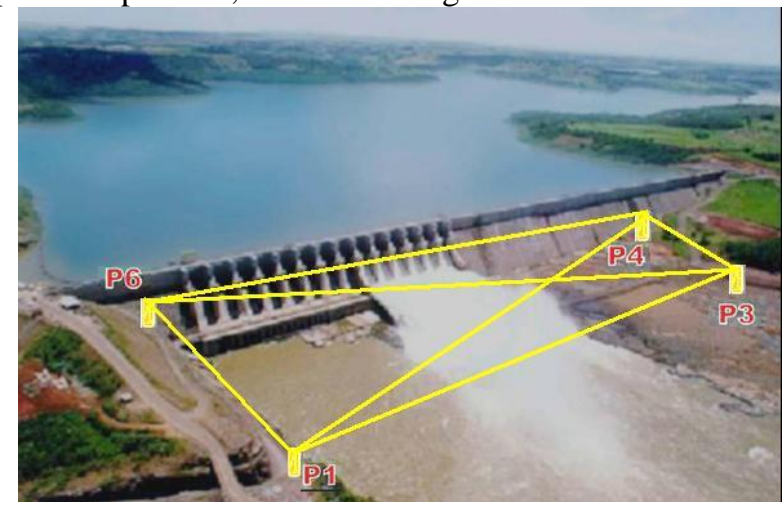

Figura 1 - Rede externa de monitoramento.

Fonte: Autor.

O monitoramento das fissuras está divido, em jusante e montante.

\subsection{Monitoramento das fissuras à jusante}

Para monitorar os pontos das fissuras localizados à jusante da barragem é necessário ocupar o pilar P3 e realizar uma visada de ré em P1 da rede externa de monitoramento, posteriormente realiza-se as visadas nos alvos implantados a direita e a esquerda das fissuras medindo-se ângulos verticais, direções horizontais e distâncias inclinadas, conforme a Fig. 2.

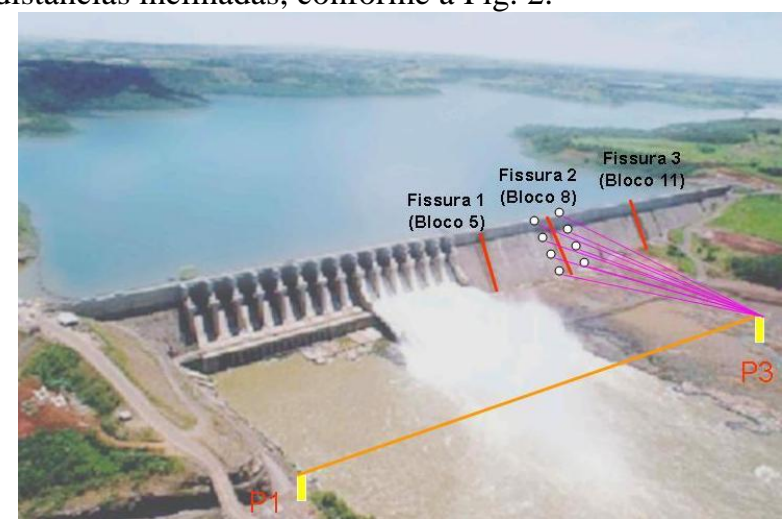

Figura 2 - Monitoramento das fissuras à jusante.

Fonte: Autor.
Foram nomeados os pontos das fissuras à jusante da barragem, onde na fissura 1 (Bloco 5) estão implantados os pontos MGE 09 a MGE 16, na fissura 2 (Bloco 8) estão implantados os pontos MGE 19 a MGE 26 e na fissura 3 (Bloco 11) estão implantados os pontos MGE 29 a MGE 36, conforme a Fig. 3.

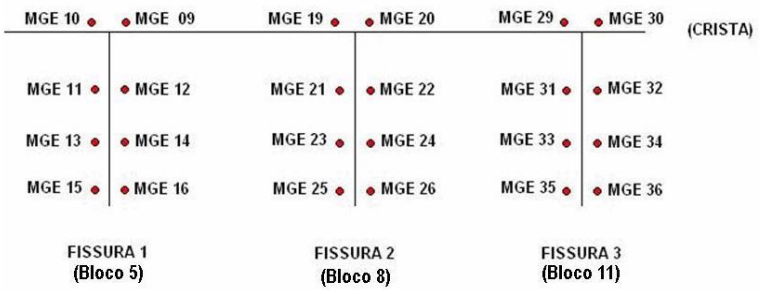

Figura 3 - Fissuras à jusante observadas a partir do pilar P3 Fonte: Autor

Visualizam-se em detalhe, na Fig. 4, os alvos localizados na fissura 1 à jusante da barragem.

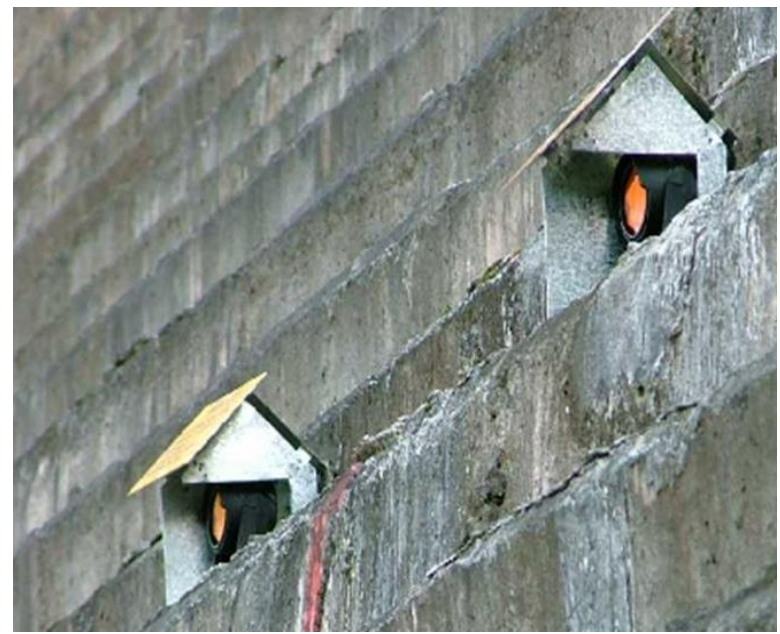

Figura 4 - Alvos à jusante da barragem.

Fonte: Autor.

\subsection{Monitoramento das fissuras à montante}

Como não é possível monitorar as fissuras a montante a partir dos pilares da rede base à jusante, implantou-se um novo ponto junto a comporta 14 definindo uma rede complementar denominada de rede externa à montante.

Para monitorar os pontos das fissuras localizados à montante, ocupa-se um vértice da rede externa à montante, localizado sobre a comporta $\mathrm{C} 14$, denominado de MGE 06 o qual tem suas coordenadas planimétricas diretamente ligadas ao referencial local adotado para a rede externa.

Foram nomeados os pontos das fissuras à montante da barragem, onde na fissura 1 (Bloco 5) estão implantados os pontos MGE 07 e MGE 08, na fissura 2 (Bloco 8) estão implantados os pontos MGE 17 e MGE 18 e na fissura 3 (Bloco 11) estão implantados os pontos MGE 27 e MGE 28, conforme Fig. 5. 


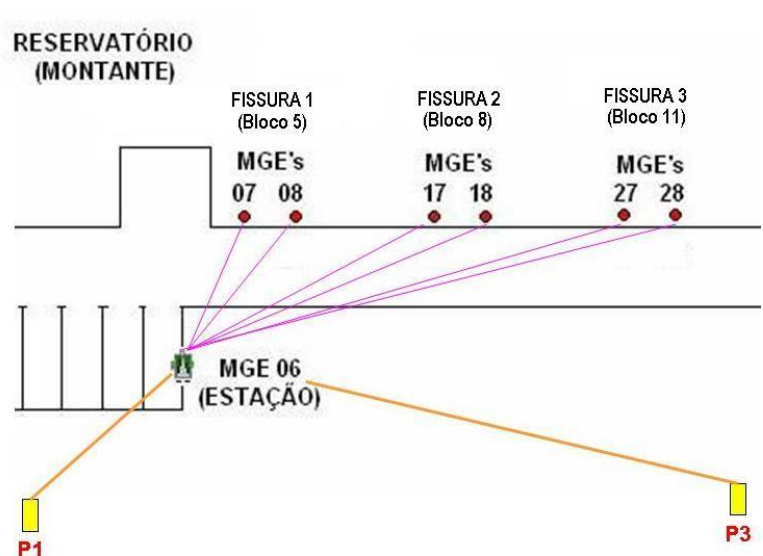

Figura 5 - Fissuras à montante observadas a partir da estação MGE 06.

Fonte: Autor.

A localização do posicionamento dos alvos à montante da barragem, (Fissura 3), sobre a mureta de proteção da pista de rolamento que encontra-se sobre à barragem, pode ser visualizada na Fig. 6.

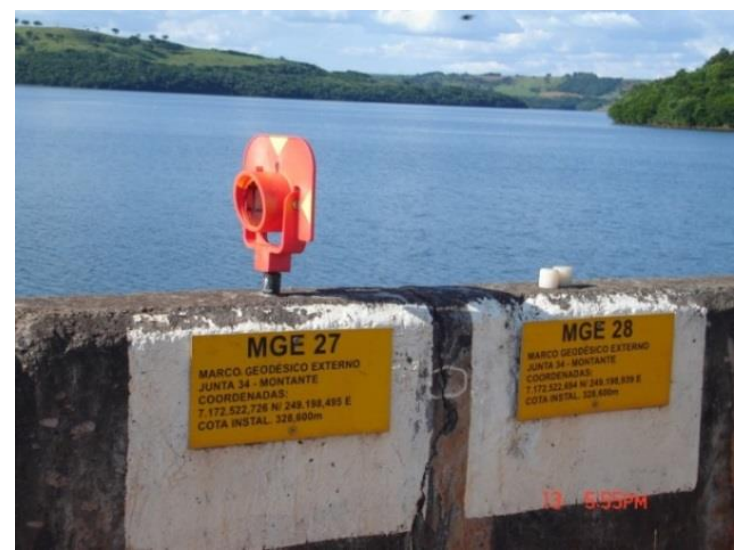

Figura 6 - Posicionamento dos alvos à montante da barragem. Fonte: Autor.

\subsection{Equipamento utilizado para o levantamento das fissuras (Blocos)}

O levantamento dos dados relacionados às fissuras foi realizado empregando-se uma estação total Robotizada Leica TCRA 1205 (Fig. 7).

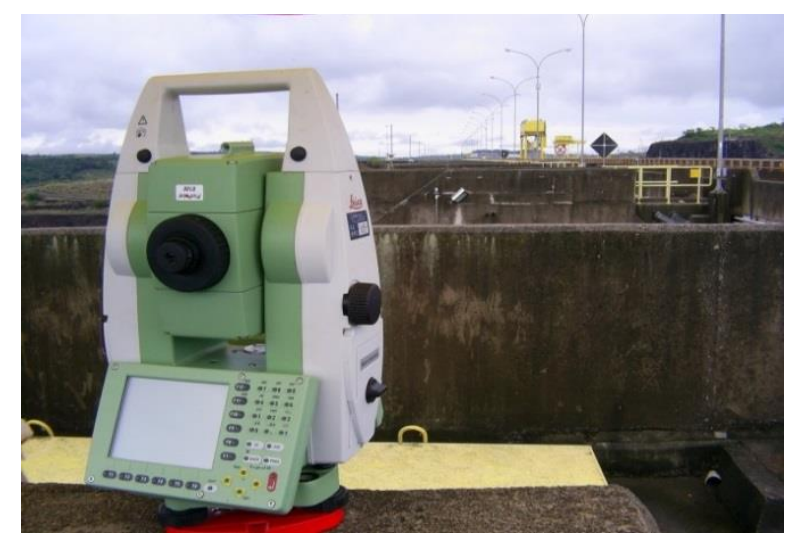

Figura 7 - Estação Total Leica TCRA 1205.

Fonte: Autor.

\section{RESULTADOS}

Foram realizadas quatro campanhas para o monitoramento das fissuras (campanhas 1, 2, 3 e 4) com o intuito de realizar análises entre épocas distintas para verificar a estabilidade dos blocos, bem como seu comportamento com a variação sazonal. As campanhas 1 e 4 foram realizadas no período da primavera e as campanhas 2 e 3 no período de inverno.

\subsection{Fissuras à jusante}

Em algumas campanhas não foi possível realizar as observações de todos os pontos, como por exemplo, na campanha 1 alguns pontos da fissura 1 estavam obstruídos por causa da vegetação próxima ao mesmo e outro foi arrancado da sua posição, permanecendo desta forma estes pontos sem informação.

As Tabs. de 1 a 12 apresentam as coordenadas das 3 fissuras (blocos) obtidas nas quatro campanhas realizadas à jusante da usina hidroelétrica de Salto Caxias.

Tabela 1 - Coordenadas da fissura 1 (bloco 5) à jusante Campanha 1

\begin{tabular}{ccccc}
\hline \multicolumn{5}{c}{ FISSURA 1 (BLOCO 5) } \\
Ponto & $\mathbf{X}(\mathbf{m})$ & $\boldsymbol{\sigma}(\mathbf{m})$ & $\mathbf{Y}(\mathbf{m})$ & $\boldsymbol{\sigma}(\mathbf{m})$ \\
\hline MGE10 & 1335,4023 & 0,0012 & 1255,8719 & 0,0012 \\
MGE09 & 1337,6386 & 0,0018 & 1255,8409 & 0,0018 \\
MGE11 & \multicolumn{2}{c}{ SEM PRISMA } & SEM PRISMA \\
MGE12 & 1336,5589 & 0,0006 & 1257,0692 & 0,0006 \\
MGE13 & SEM LEITURA & SEM LEITURA \\
MGE14 & SEM LEITURA & SEM LEITURA \\
MGE15 & SEM LEITURA & SEM LEITURA \\
MGE16 & 1337,5999 & 0,0017 & 1237,2682 & 0,0016 \\
\hline
\end{tabular}

Tabela 2 - Coordenadas da fissura 2 (bloco 8) à jusante Campanha 1

\begin{tabular}{ccccl}
\hline & \multicolumn{5}{c}{ FISSURA 2 (BLOCO 8) } \\
Ponto & $\mathbf{X}(\mathbf{m})$ & $\boldsymbol{\sigma}(\mathbf{m})$ & $\mathbf{Y}(\mathbf{m})$ & $\boldsymbol{\sigma}(\mathbf{m})$ \\
\hline MGE19 & 1466,4830 & 0,0011 & 1253,7382 & 0,0023 \\
MGE20 & 1469,3029 & 0,0011 & 1253,6985 & 0,0023 \\
MGE21 & 1467,7103 & 0,0010 & 1254,9298 & 0,0023 \\
MGE22 & 1469,5273 & 0,0010 & 1254,9152 & 0,0022 \\
MGE23 & 1467,2854 & 0,0004 & 1247,2963 & 0,0005 \\
MGE24 & 1469,0008 & 0,0003 & 1247,2763 & 0,0005 \\
MGE25 & 1467,0465 & 0,0003 & 1236,9486 & 0,0005 \\
MGE26 & 1468,3374 & 0,0003 & 1236,9341 & 0,0005 \\
\hline
\end{tabular}

Tabela 3 - Coordenadas da fissura 3 (bloco 11) à jusante Campanha 1

\begin{tabular}{ccccc}
\hline \multicolumn{5}{c}{ FISSURA 3 (BLOCO 11) } \\
Ponto & $\mathbf{X}(\mathbf{m})$ & $\boldsymbol{\sigma}(\mathbf{m})$ & $\mathbf{Y}(\mathbf{m})$ & $\boldsymbol{\sigma}(\mathbf{m})$ \\
\hline MGE29 & 1578,3108 & 0,0004 & 1251,9119 & 0,0011 \\
MGE30 & 1579,6242 & 0,0003 & 1251,9089 & 0,0010 \\
MGE31 & 1577,9731 & 0,0003 & 1253,1375 & 0,0011 \\
MGE32 & 1579,4794 & 0,0002 & 1253,1141 & 0,0011 \\
MGE33 & 1577,3451 & 0,0003 & 1245,2927 & 0,0012 \\
MGE34 & 1578,6183 & 0,0004 & 1245,2726 & 0,0010 \\
MGE35 & 1576,5921 & 0,0003 & 1233,5778 & 0,0009 \\
MGE36 & 1577,5393 & 0,0004 & 1233,5811 & 0,0007 \\
\hline
\end{tabular}


Tabela 4 - Coordenadas da fissura 1 (bloco 5) à jusante Campanha 2

\begin{tabular}{ccccc}
\hline \multicolumn{5}{c}{ FISSURA 1 (BLOCO 5) } \\
Ponto & $\mathbf{X}(\mathbf{m})$ & $\boldsymbol{\sigma}(\mathbf{m})$ & $\mathbf{Y}(\mathbf{m})$ & $\boldsymbol{\sigma}(\mathbf{m})$ \\
\hline MGE10 & 1335,4025 & 0,0024 & 1255,8757 & 0,0024 \\
MGE09 & 1337,6385 & 0,0028 & 1255,8439 & 0,0029 \\
MGE11 & \multicolumn{2}{c}{ SEM PRISMA } & \multicolumn{2}{c}{ SEM PRISMA } \\
MGE12 & 1336,5591 & 0,0014 & 1257,0714 & 0,0015 \\
MGE13 & \multicolumn{2}{c}{ SEM LEITURA } & \multicolumn{2}{c}{ SEM LEITURA } \\
MGE14 & 1336,3887 & 0,0010 & 1247,1604 & 0,0011 \\
MGE15 & \multicolumn{2}{c}{ SEM LEITURA } & SEM LEITURA \\
MGE16 & 1337,6065 & 0,0011 & 1237,2723 & 0,0011 \\
\hline
\end{tabular}

Tabela 5 - Coordenadas da fissura 2 (bloco 8) à jusante Campanha 2

\begin{tabular}{ccccc}
\hline & \multicolumn{4}{c}{ FISSURA 2 (BLOCO 8) } \\
Ponto & $\mathbf{X}(\mathbf{m})$ & $\boldsymbol{\sigma}(\mathbf{m})$ & $\mathbf{Y}(\mathbf{m})$ & $\boldsymbol{\sigma}(\mathbf{m})$ \\
\hline MGE19 & 1466,4752 & 0,0014 & 1253,7412 & 0,0029 \\
MGE20 & 1469,2971 & 0,0012 & 1253,7020 & 0,0027 \\
MGE21 & 1467,7025 & 0,0012 & 1254,9303 & 0,0025 \\
MGE22 & 1469,5200 & 0,0012 & 1254,9167 & 0,0028 \\
MGE23 & 1467,2796 & 0,0005 & 1247,2965 & 0,0007 \\
MGE24 & 1468,9932 & 0,0004 & 1247,2766 & 0,0007 \\
MGE25 & 1467,0421 & 0,0004 & 1236,9488 & 0,0006 \\
MGE26 & 1468,3318 & 0,0004 & 1236,9343 & 0,0008 \\
\hline
\end{tabular}

Tabela 6 - Coordenadas da fissura 3 (bloco 11) à jusante Campanha 2

\begin{tabular}{ccccc}
\hline & \multicolumn{4}{c}{ FISSURA 3 (BLOCO 11) } \\
Ponto & $\mathbf{X}(\mathbf{m})$ & $\boldsymbol{\sigma}(\mathbf{m})$ & $\mathbf{Y}(\mathbf{m})$ & $\boldsymbol{\sigma}(\mathbf{m})$ \\
\hline MGE29 & 1578,3139 & 0,0005 & 1251,9090 & 0,0016 \\
MGE30 & 1579,6265 & 0,0007 & 1251,9061 & 0,0016 \\
MGE31 & 1577,9742 & 0,0005 & 1253,1340 & 0,0017 \\
MGE32 & 1579,4825 & 0,0005 & 1253,1105 & 0,0014 \\
MGE33 & 1577,3492 & 0,0005 & 1245,2881 & 0,0016 \\
MGE34 & 1578,6205 & 0,0003 & 1245,2676 & 0,0014 \\
MGE35 & 1576,5953 & 0,0002 & 1233,5723 & 0,0011 \\
MGE36 & 1577,5396 & 0,0003 & 1233,5757 & 0,0010 \\
\hline
\end{tabular}

Tabela 7 - Coordenadas da fissura 1 (bloco 5) à jusante Campanha 3

\begin{tabular}{ccccc}
\hline \multicolumn{5}{c}{ FISSURA 1 (BLOCO 5) } \\
Ponto & $\mathbf{X}(\mathbf{m})$ & $\boldsymbol{\sigma}(\mathbf{m})$ & $\mathbf{Y}(\mathbf{m})$ & $\boldsymbol{\sigma}(\mathbf{m})$ \\
\hline MGE10 & 1335,4021 & 0,0012 & 1255,8755 & 0,0012 \\
MGE09 & 1337,6402 & 0,0015 & 1255,8436 & 0,0016 \\
MGE11 & SEM PRISMA & \multicolumn{3}{c}{ SEM PRISMA } \\
MGE12 & 1336,5592 & 0,0009 & 1257,0723 & 0,0009 \\
MGE13 & 1330,1807 & 0,0005 & 1247,2809 & 0,0010 \\
MGE14 & 1336,3840 & 0,0011 & 1247,1688 & 0,0011 \\
MGE15 & 1329,7859 & 0,0016 & 1237,4179 & 0,0015 \\
MGE16 & 1337,6019 & 0,0018 & 1237,2712 & 0,0017 \\
\hline
\end{tabular}

Tabela 8 - Coordenadas da fissura 2 (bloco 8) à jusante Campanha 3

\begin{tabular}{ccccc}
\hline \multicolumn{5}{c}{ FISSURA 2 (BLOCO 8) } \\
Ponto & $\mathbf{X}(\mathbf{m})$ & $\boldsymbol{\sigma}(\mathbf{m})$ & $\mathbf{Y}(\mathbf{m})$ & $\boldsymbol{\sigma}(\mathbf{m})$ \\
\hline MGE19 & 1466,4842 & 0,0007 & 1253,7425 & 0,0016 \\
MGE20 & 1469,3041 & 0,0006 & 1253,7031 & 0,0013 \\
MGE21 & 1467,7109 & 0,0007 & 1254,9322 & 0,0014 \\
MGE22 & 1469,5268 & 0,0006 & 1254,9180 & 0,0014 \\
MGE23 & 1467,2872 & 0,0002 & 1247,3001 & 0,0003 \\
MGE24 & 1469,0020 & 0,0003 & 1247,2789 & 0,0003 \\
MGE25 & 1467,0488 & 0,0002 & 1236,9519 & 0,0003 \\
MGE26 & 1468,3395 & 0,0002 & 1236,9369 & 0,0003 \\
\hline
\end{tabular}

Tabela 9 - Coordenadas da fissura 3 (bloco 11) à jusante Campanha 3

\begin{tabular}{ccccc}
\hline \multicolumn{5}{c}{ FISSURA 3 (BLOCO 11) } \\
Ponto & $\mathbf{X}(\mathbf{m})$ & $\boldsymbol{\sigma}(\mathbf{m})$ & $\mathbf{Y}(\mathbf{m})$ & $\boldsymbol{\sigma}(\mathbf{m})$ \\
\hline MGE29 & 1578,3129 & 0,0002 & 1251,9068 & 0,0007 \\
MGE30 & 1579,6243 & 0,0002 & 1251,9040 & 0,0007 \\
MGE31 & 1577,9720 & 0,0003 & 1253,1318 & 0,0010 \\
MGE32 & 1579,4807 & 0,0003 & 1253,1084 & 0,0006 \\
MGE33 & 1577,3458 & 0,0001 & 1245,2876 & 0,0006 \\
MGE34 & 1578,6184 & 0,0001 & 1245,2668 & 0,0009 \\
MGE35 & 1576,5925 & 0,0001 & 1233,5723 & 0,0004 \\
MGE36 & 1577,5391 & 0,0002 & 1233,5754 & 0,0005 \\
\hline
\end{tabular}

Tabela 10 - Coordenadas da fissura 1 (bloco 05) à jusante Campanha 4

\begin{tabular}{ccccc}
\hline \multicolumn{5}{c}{ FISSURA 1 (BLOCO 5) } \\
Ponto & $\mathbf{X}(\mathbf{m})$ & $\boldsymbol{\sigma}(\mathbf{m})$ & $\mathbf{Y}(\mathbf{m})$ & $\boldsymbol{\sigma}(\mathbf{m})$ \\
\hline MGE10 & 1335,4057 & 0,0016 & 1255,8708 & 0,0017 \\
MGE09 & 1337,6431 & 0,0021 & 1255,8402 & 0,0022 \\
MGE11 & \multicolumn{2}{c}{ SEM PRISMA } & SEM PRISMA \\
MGE12 & 1336,5634 & 0,0008 & 1257,0667 & 0,0009 \\
MGE13 & 1330,1857 & 0,0006 & 1247,2771 & 0,0006 \\
MGE14 & 1330,1848 & 0,0008 & 1247,2760 & 0,0008 \\
MGE15 & SEM PRISMA & SEM PRISMA \\
MGE16 & SEM PRISMA & SEM PRISMA \\
\hline
\end{tabular}

Tabela 11 - Coordenadas da fissura 2 (bloco 8) à jusante Campanha 4

\begin{tabular}{ccccc}
\hline \multicolumn{5}{c}{ FISSURA 2 (BLOCO 8) } \\
Ponto & $\mathbf{X}(\mathbf{m})$ & $\boldsymbol{\sigma}(\mathbf{m})$ & $\mathbf{Y}(\mathbf{m})$ & $\boldsymbol{\sigma}(\mathbf{m})$ \\
\hline MGE19 & 1466,4810 & 0,0007 & 1253,7366 & 0,0014 \\
MGE20 & 1469,3002 & 0,0009 & 1253,6981 & 0,0019 \\
MGE21 & 1467,7073 & 0,0007 & 1254,9263 & 0,0015 \\
MGE22 & 1469,5239 & 0,0006 & 1254,9122 & 0,0013 \\
MGE23 & 1467,2817 & 0,0002 & 1247,2930 & 0,0003 \\
MGE24 & 1468,9973 & 0,0001 & 1247,2720 & 0,0002 \\
MGE25 & 1467,0432 & 0,0001 & 1236,9439 & 0,0002 \\
MGE26 & 1468,3332 & 0,0003 & 1236,9294 & 0,0003 \\
\hline
\end{tabular}

Tabela 12 - Coordenadas da fissura 3 (bloco 11) à jusante Campanha 4

\begin{tabular}{ccccc}
\hline \multicolumn{5}{c}{ FISSURA 3 (BLOCO 11) } \\
Ponto & $\mathbf{X}(\mathbf{m})$ & $\boldsymbol{\sigma}(\mathbf{m})$ & $\mathbf{Y}(\mathbf{m})$ & $\boldsymbol{\sigma}(\mathbf{m})$ \\
\hline MGE29 & 1578,3132 & 0,0004 & 1251,9046 & 0,0008 \\
MGE30 & 1579,6265 & 0,0002 & 1251,9010 & 0,0007 \\
MGE31 & 1577,9746 & 0,0004 & 1253,1287 & 0,0017 \\
MGE32 & 1579,4829 & 0,0004 & 1253,1046 & 0,0006 \\
MGE33 & 1577,3475 & 0,0001 & 1245,2837 & 0,0006 \\
MGE34 & 1578,6212 & 0,0001 & 1245,2630 & 0,0006 \\
MGE35 & 1576,5936 & 0,0001 & 1233,5687 & 0,0004 \\
MGE36 & 1577,5418 & 0,0001 & 1233,5710 & 0,0004 \\
\hline
\end{tabular}

\subsubsection{Interpretação dos deslocamentos à jusante}

A análise das discrepâncias das coordenadas dos pontos das fissuras à jusante foi realizada com base na comparação entre as campanhas, sendo estas comparações realizadas duas a duas (entre as campanhas 1 e 4 período de primavera e as campanhas 2 e 3 - período de inverno). Este critério foi adotado com o intuito de analisar também, as coordenadas em épocas com variações sazonais. 
$\mathrm{Na}$ Tab. 13 são apresentadas as variação das coordenadas das fissuras à jusante entre as campanhas $1 \mathrm{e}$ 4.

Tabela 13 - Variação das coordenadas das fissuras à jusante Campanhas 1 e 4

\begin{tabular}{ccc}
\hline \multicolumn{3}{c}{ Fissura1 } \\
Ponto & X(m) & Y(m) \\
\hline MGE10 & $-0,0034$ & 0,0011 \\
MGE09 & $-0,0045$ & 0,0007 \\
MGE11 & SEM PRISMA \\
MGE12 & $-0,0045$ & 0,0025 \\
MGE13 & SEM LEITURA \\
MGE14 & SEM LEITURA \\
MGE15 & SEM LEITURA \\
MGE16 & SEM LEITURA \\
\hline \multicolumn{3}{c}{ Fissura2 } \\
Ponto & X(m) & Y(m) \\
\hline MGE19 & 0,0020 & 0,0016 \\
MGE20 & 0,0027 & 0,0004 \\
MGE21 & 0,0030 & 0,0035 \\
MGE22 & 0,0034 & 0,0030 \\
MGE23 & 0,0037 & 0,0033 \\
MGE24 & 0,0035 & 0,0043 \\
MGE25 & 0,0033 & 0,0047 \\
MGE26 & 0,0042 & 0,0047 \\
\hline \multicolumn{3}{c}{ Fissura3 } \\
Ponto & X(m) & Y(m) \\
\hline MGE29 & $-0,0024$ & 0,0073 \\
MGE30 & $-0,0023$ & 0,0079 \\
MGE31 & $-0,0015$ & 0,0088 \\
MGE32 & $-0,0035$ & 0,0095 \\
MGE33 & $-0,0024$ & 0,0090 \\
MGE34 & $-0,0029$ & 0,0096 \\
MGE35 & $-0,0015$ & 0,0091 \\
MGE36 & $-0,0025$ & 0,0101 \\
\hline & \multicolumn{2}{c}{}
\end{tabular}

Nas Figs. 8, 9 e 10 são representados os gráficos de variação das coordenadas das fissuras 1, 2 e 3 à jusante pertinentes à Tab. 13 .

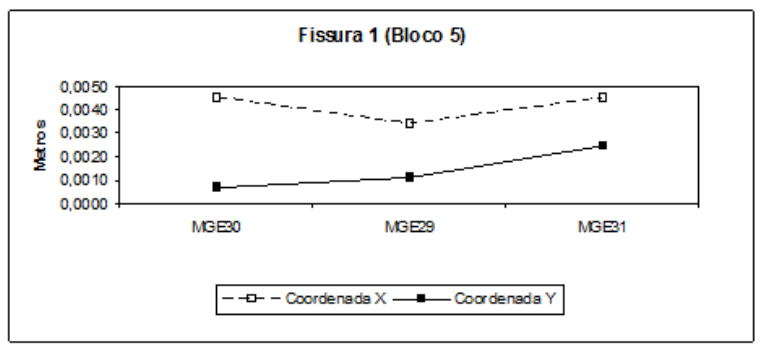

Figura 8 - Variação das coordenadas da fissura 1 à jusante. Fonte: Autor.

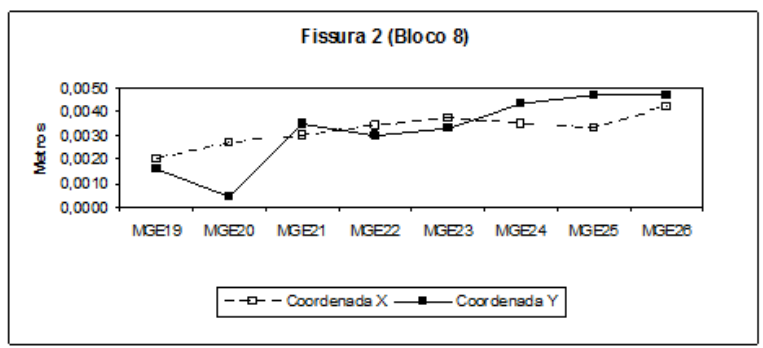

Figura 9 - Variação das coordenadas da fissura 2 à jusante. Fonte: Autor.

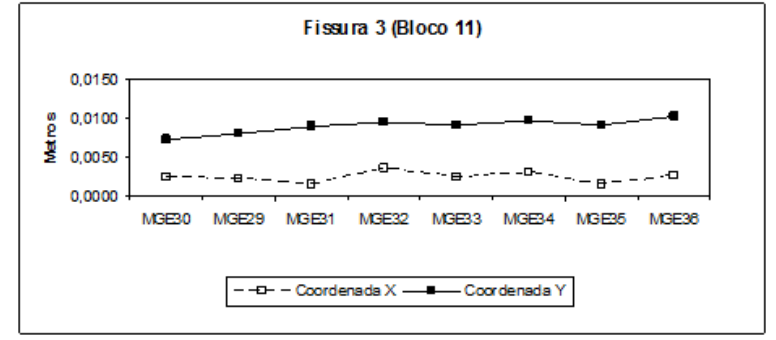

Figura 10 - Variação das coordenadas da fissura 3 à jusante. Fonte: Autor.

$\mathrm{Na}$ Tab. 14 são apresentadas as variação das coordenadas das fissuras à jusante entre as campanhas $2 \mathrm{e}$ 3.

Tabela 14 - Variação das coordenadas das fissuras à jusante Campanhas 2 e 3

\begin{tabular}{ccc}
\hline \multicolumn{3}{c}{ Fissura1 } \\
Ponto & X(m) & Y(m) \\
\hline MGE10 & $-0,0017$ & 0,0003 \\
MGE09 & 0,0004 & 0,0002 \\
MGE11 & SEM PRISMA \\
MGE12 & $-0,0001$ & $-0,0009$ \\
MGE13 & SEM LEITURA \\
MGE14 & 0,0047 & $-0,0084$ \\
MGE15 & SEM LEITURA \\
MGE16 & 0,0046 & 0,0011 \\
\hline \multicolumn{3}{c}{ Fissura2 } \\
Ponto & X(m) & Y(m) \\
\hline MGE19 & $-0,0070$ & $-0,0011$ \\
MGE20 & $-0,0090$ & $-0,0013$ \\
MGE21 & $-0,0084$ & $-0,0019$ \\
MGE22 & $-0,0068$ & $-0,0013$ \\
MGE23 & $-0,0076$ & $-0,0036$ \\
MGE24 & $-0,0088$ & $-0,0023$ \\
MGE25 & $-0,0067$ & $-0,0031$ \\
MGE26 & $-0,0077$ & $-0,0026$ \\
\hline \multicolumn{3}{c}{ Fissura3 } \\
Ponto & X(m) & Y(m) \\
\hline MGE29 & 0,0022 & 0,0021 \\
MGE30 & 0,0010 & 0,0022 \\
MGE31 & 0,0022 & 0,0022 \\
MGE32 & 0,0018 & 0,0021 \\
MGE33 & 0,0034 & 0,0005 \\
MGE34 & 0,0021 & 0,0008 \\
MGE35 & 0,0028 & 0,0000 \\
MGE36 & 0,0005 & 0,0003 \\
\hline
\end{tabular}

Nas Figs. 11, 12 e 13 são representados os gráficos de variação das coordenadas das fissuras 1,2 e 3 à jusante pertinentes à Tab. 14.

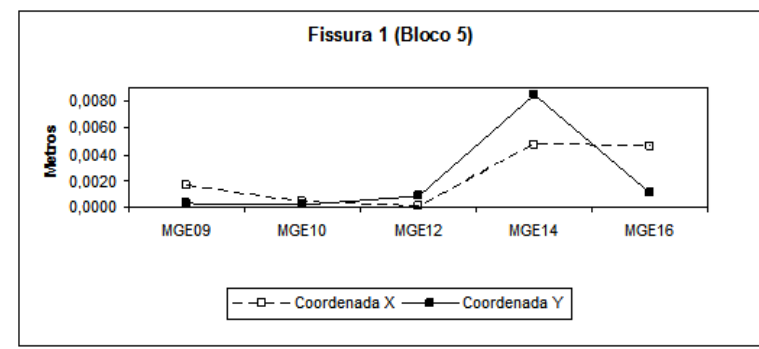

Figura 11 - Variação das coordenadas da fissura 1 à jusante. Fonte: Autor. 


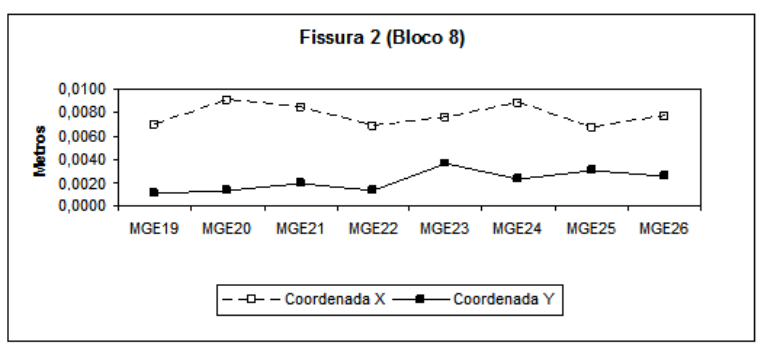

Figura 12 - Variação das coordenadas da fissura 2 à jusante. Fonte: Autor.

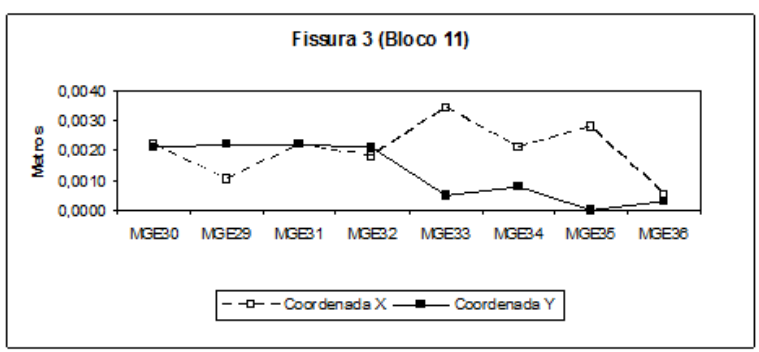

Figura 13 - Variação das coordenadas da fissura 3 à jusante. Fonte: Autor.

\subsubsection{Teste estatístico e interpretações dos deslocamentos à jusante}

Obtidas as coordenadas de todas as fissuras (Blocos 5, Bloco 8 e Bloco 11), calcularam-se as diferenças entre duas épocas, (entre as campanhas 1 e 4 período de primavera e entre as campanhas 2 e 3 - período de inverno). Para verificar se estas variações são significativas aplicou-se o teste estatístico de Snedecor com distribuição $F$, a um nível de significância de $5 \%$. Neste teste a hipótese básica é dada pela Eq.1.

$$
H_{0}: \tilde{d}=\left[\begin{array}{l}
d x \\
d y
\end{array}\right]=\left[\begin{array}{l}
0 \\
0
\end{array}\right]
$$

onde não existe diferença significativa entre as componentes. Uma hipótese alternativa é dada pela Eq.2.

$$
H_{0}: \tilde{d}=\left[\begin{array}{l}
d x \\
d y
\end{array}\right] \neq\left[\begin{array}{l}
0 \\
0
\end{array}\right]
$$

onde existe diferença significativa entre as componentes.

Na hipótese básica e alternativa tem-se:

$\mathrm{d}_{\mathrm{x}}=$ vetor da componente $\mathrm{x}$, resultante da diferença entre duas épocas;

$\mathrm{d}_{\mathrm{y}}=$ vetor da componente $\mathrm{y}$, resultante da diferença entre duas épocas dada pela Eq.3.

$$
\tilde{d}=\left[\begin{array}{l}
d x \\
d y
\end{array}\right]
$$

O vetor médio das diferenças entre componentes é dada pela Eq.4.

$$
\begin{aligned}
& \tilde{d}=n^{-1} \cdot \sum_{j=1}^{n} \tilde{d}_{j} \\
& S_{d}=n^{-1} \cdot \sum_{j=1}^{n} \tilde{d}_{j}(\widetilde{d} J-d)\left(\tilde{d}_{j}-d\right)^{T}
\end{aligned}
$$

Estatística do teste:

$$
V C=n \cdot\left(d-\widetilde{d)^{T}} \cdot\left(S_{d}\right)^{-1} \cdot\left(d_{j}-\tilde{d}\right)\right.
$$

Como:

$$
V T=(p(n-1) / n-p) \cdot F(\alpha)_{p, n-p}
$$

Sendo:

$n=$ número de pontos;

$p=$ número de variáveis

Rejeita-se $\mathrm{H}_{0}$, se:

$$
V C>(p(n-1) / n-p) \cdot F(\alpha)_{p, n-p}
$$

Com base nas hipóteses acima, a seguir são apresentados os resultados dos cálculos do teste estatístico entre as duas comparações à jusante:

$\checkmark \quad$ Resultado entre as Campanhas 1 e 2

Os resultados foram:

- Valor Teórico (VT), conforme Eq.7.

$$
\begin{aligned}
& p(n-1) /(n-p) \cdot F(\alpha)_{p, n-p}= \\
& =\left(2(8-1) /(8-2) \cdot F(0,05)_{2,6}=3,8\right.
\end{aligned}
$$

- Valor Calculado (VC), conforme Eq.8.

$$
V C=n \cdot(d-\widetilde{d})^{T} \cdot\left(S_{d}\right)^{-1} \cdot\left(d_{j}-\tilde{d}\right)=2,3
$$

Logo:

$$
\mathrm{VC}=2,3<V T=3,8
$$

E assim sendo, aceita-se a hipótese básica $\mathrm{H}_{0}$ a um nível de significância de 5\%, cujo resultado indica que não existe diferença significativa entre os valores obtidos.

\section{Resultado entre as Campanhas 3 e 4}

Os resultados foram:

- Valor Teórico (VT), conforme Eq.7.

$p(n-1) /(n-p) \cdot F(\alpha)_{p, n-p}=$ 
$=\left(2(8-1) /(8-2) \cdot F(0,05)_{2,6}=3,8\right.$

Valor Calculado (VC), conforme Eq.8.

$$
V C=n \cdot\left(d-\widetilde{d)^{T}} \cdot\left(S_{d}\right)^{-1} \cdot\left(d_{j}-\tilde{d}\right)=2,0\right.
$$

Logo:

$$
\mathrm{VC}=2,0<V T=3,8
$$

E assim sendo, aceita-se a hipótese básica $\mathrm{H}_{0}$ a um nível de significância de 5\%, cujo resultado indica que não existe diferença significativa entre os valores obtidos.

\subsection{Fissuras à montante}

Nas Tabs. 15, 16 e 17 são apresentadas as coordenadas dos pontos das fissuras (blocos), obtidas nas quatro campanhas realizadas à montante da usina hidroelétrica de Salto Caxias.

Tabela 15 - Coordenadas da fissura 1 (Bloco 5) à montante Todas as épocas

\begin{tabular}{ccccc}
\hline \multicolumn{5}{c}{ FISSURA I (BLOCO 5) } \\
Ponto & X(m) & $\sigma(\mathbf{m})$ & Y(m) & $\sigma(\mathbf{m})$ \\
\hline \multicolumn{5}{c}{ CAMPANHA 1 } \\
\hline MGE07 & 1337,8153 & 0,0002 & 1264,5621 & 0,0002 \\
MGE08 & 1338,5323 & 0,0002 & 1264,5574 & 0,0002 \\
\hline \multicolumn{5}{c}{ CAMPANHA 2 } \\
\hline MGE07 & 1337,8158 & 0,0002 & 1264,5625 & 0,0001 \\
MGE08 & 1338,5330 & 0,0001 & 1264,5581 & 0,0001 \\
\hline \multicolumn{5}{c}{ CAMPANHA 3 } \\
\hline MGE07 & 1337,8147 & 0,0001 & 1264,5617 & 0,0001 \\
MGE08 & 1338,5310 & 0,0001 & 1264,5569 & 0,0001 \\
\hline \multicolumn{5}{c}{ CAMPANHA 4 } \\
\hline MGE07 & 1337,8151 & 0,0001 \\
MGE08 & 1338,5313 & 0,0001 & 1264,5615 & 0,0001 \\
\hline
\end{tabular}

Tabela 16 - Coordenadas da fissura 2 (Bloco 8) à montante -

\begin{tabular}{|c|c|c|c|c|}
\hline \multirow[b]{2}{*}{ Ponto } & \multicolumn{3}{|c|}{ FISSURA 2 (BLOCO 8) } & \multirow[b]{2}{*}{$\sigma(\mathbf{m})$} \\
\hline & $\mathbf{X}(\mathbf{m})$ & $\sigma(\mathbf{m})$ & $\mathbf{Y}(\mathbf{m})$ & \\
\hline \multicolumn{5}{|c|}{ CAMPANHA 1} \\
\hline MGE17 & 1467,3852 & 0,0023 & 1262,4235 & 0,0005 \\
\hline MGE18 & 1467,7265 & 0,0019 & 1262,4099 & 0,0003 \\
\hline \multicolumn{5}{|c|}{ CAMPANHA 2} \\
\hline MGE17 & 1467,3858 & 0,0008 & 1262,4294 & 0,0006 \\
\hline MGE18 & 1467,7287 & 0,0018 & 1262,4123 & 0,0002 \\
\hline \multicolumn{5}{|c|}{ CAMPANHA 3} \\
\hline MGE17 & 1467,3853 & 0,0007 & 1262,4242 & 0,0002 \\
\hline MGE18 & 1467,7261 & 0,0006 & 1262,4111 & 0,0001 \\
\hline \multicolumn{5}{|c|}{ CAMPANHA 4} \\
\hline MGE17 & 1467,3883 & 0,0008 & 1262,4258 & 0,0003 \\
\hline MGE18 & 1467,7270 & 0,0009 & 1262,4112 & 0,0002 \\
\hline
\end{tabular}
Todas as épocas

Figura 14 - Variação das coordenadas da fissura 1 à montante.

Fonte: Autor.
Tabela 17 - Coordenadas da fissura 3 (Bloco 11) à montante Todas as épocas

\begin{tabular}{ccccc}
\hline \multicolumn{5}{c}{ FISSURA 1 (BLOCO 5) } \\
Ponto & X(m) & $\sigma(\mathbf{m})$ & Y(m) & $\boldsymbol{\sigma}(\mathbf{m})$ \\
\hline \multicolumn{5}{c}{ CAMPANHA 1 } \\
MGE27 & 1579,1570 & 0,0001 & 1260,6128 & 0,0019 \\
MGE28 & 1579,5971 & 0,0034 & 1260,6048 & 0,0003 \\
\hline \multicolumn{5}{c}{ CAMPANHA 2 } \\
\hline MGE27 & 1579,1594 & 0,0024 & 1260,6179 & 0,0008 \\
MGE28 & 1579,6002 & 0,0025 & 1260,6074 & 0,0002 \\
\hline \multicolumn{5}{c}{ CAMPANHA 3 } \\
\hline MGE27 & 1579,1571 & 0,0014 \\
MGE28 & 1579,5977 & 0,0012 & 1260,6129 & 0,0002 \\
\hline \multicolumn{5}{c}{ CAMPANHA 4 } \\
\hline MGE27 & 1579,1566 & 0,0014 \\
MGE28 & 1579,5978 & 0,0013 & 1260,6147 & 0,0002 \\
\hline \multicolumn{5}{c}{1260,6045} \\
\hline
\end{tabular}

\subsubsection{Interpretação dos deslocamentos à montante}

A variação das coordenadas dos pontos das fissuras à montante também foi analisado realizando a comparação entre dados de levantamentos de duas épocas distintas. A primeira análise foi realizada entre as campanhas 1 e 4 e uma segunda análise entre as campanhas 2 e 3, objetivando desta maneira simular épocas com variações sazonais.

$\mathrm{Na}$ Tab. 18 são apresentas as variações das coordenadas das fissuras à montante entre as campanhas 1 e 4 .

Tabela 18 - Variação das coordenadas das fissuras à montante Campanha 1 e 4

\begin{tabular}{cll}
\hline & Fissura 1 \\
Ponto & X(m) & Y(m) \\
\hline MGE07 & 0,0002 & 0,0006 \\
MGE08 & 0,0010 & 0,0006 \\
\hline & Fissura 2 \\
Ponto & X(m) & $\mathbf{Y}(\mathbf{m})$ \\
\hline MGE17 & $-0,0031$ & $-0,0023$ \\
MGE18 & $-0,0005$ & $-0,0013$ \\
\hline & Fissura 3 \\
Ponto & $\mathbf{X}(\mathbf{m})$ & $\mathbf{Y}(\mathbf{m})$ \\
\hline MGE27 & 0,0004 & $-0,0019$ \\
MGE28 & $-0,0007$ & 0,0003 \\
\hline
\end{tabular}

Nas Figs. 14, 15 e 16 são representados os gráficos de variação das coordenadas das fissuras 1,2 e 3 à montante pertinentes à Tab. 18.






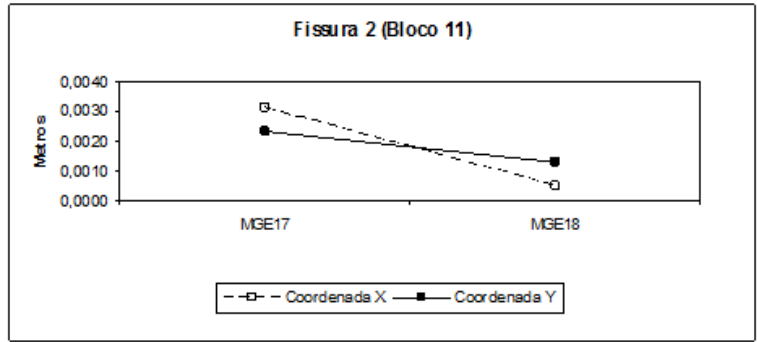

Figura 15 - Variação das coordenadas da fissura 2 à montante. Fonte: Autor.

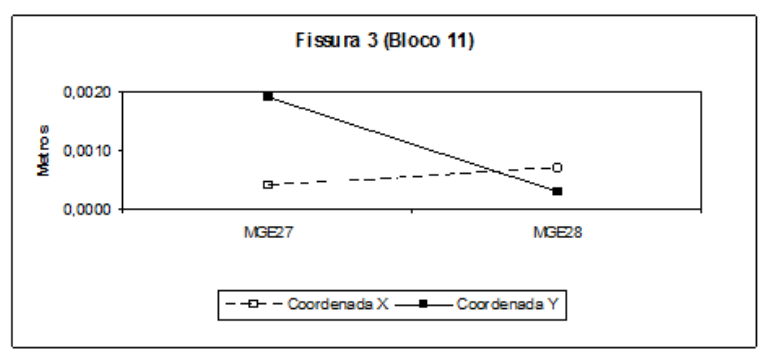

Figura 16 - Variação das coordenadas da fissura 3 à montante Fonte: Autor.

Na Tab. 19 são apresentas as variações das coordenadas das fissuras à montante, porém agora entre as campanhas 2 e 3 na Tab. 19.

Tabela 19 - Variação das coordenadas das fissuras à montante Campanhas 2 e 3

\begin{tabular}{cll}
\hline & Fissura 1 \\
Ponto & X(m) & Y(m) \\
\hline MGE07 & 0,0002 & 0,0006 \\
MGE08 & 0,0010 & 0,0006 \\
\hline & Fissura 2 \\
Ponto & X(m) & $\mathbf{Y}(\mathbf{m})$ \\
\hline MGE17 & $-0,0031$ & $-0,0023$ \\
MGE18 & $-0,0005$ & $-0,0013$ \\
\hline & Fissura 3 & \\
Ponto & $\mathbf{X}(\mathbf{m})$ & $\mathbf{Y}(\mathbf{m})$ \\
\hline MGE27 & 0,0004 & $-0,0019$ \\
MGE28 & $-0,0007$ & 0,0003 \\
\hline
\end{tabular}

As Figs. 17, 18 e 19 representam os gráficos de variação das coordenadas das fissuras 1,2 e 3 à montante pertinentes à Tab. 19 .

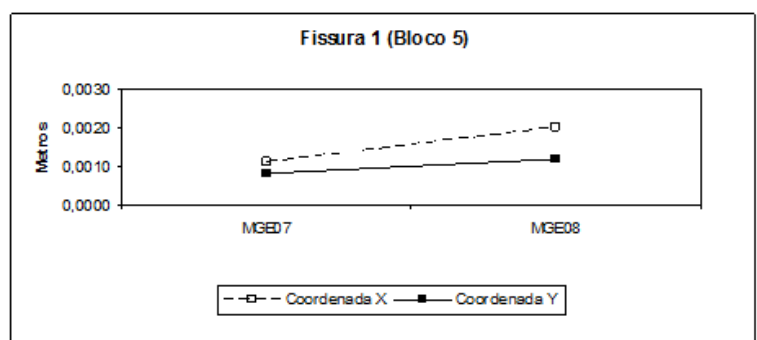

Figura 17 - Variação das coordenadas da fissura 1 à montante. Fonte: Autor.

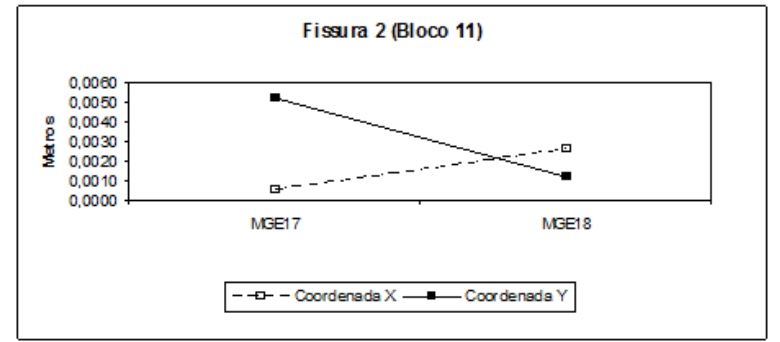

Figura 18 - Variação das coordenadas da fissura 2 à montante. Fonte: Autor.

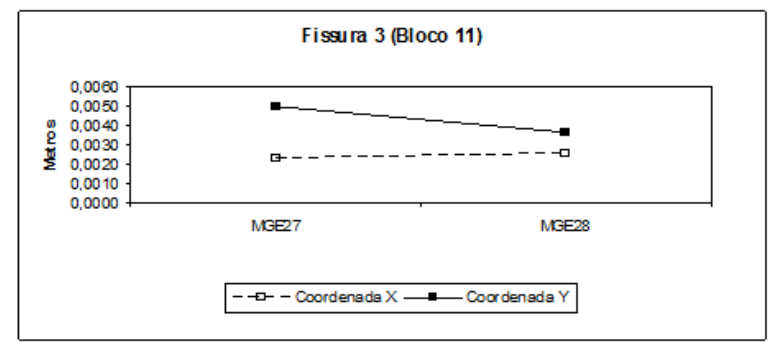

Figura 19 - Variação das coordenadas da fissura 3 à montante. Fonte: Autor.

\subsubsection{Teste estatístico e interpretações dos deslocamentos à montante}

Da mesma maneira que à jusante calcularam-se as diferenças entre as campanhas para à montante e foram aplicados os testes estatísticos para a validação destes dados.

A seguir são apresentados os resultados entre as duas comparações à montante.

$\checkmark \quad$ Resultados entre as Campanha 1 e 4

Os resultados obtidos foram:

- Valor Teórico (VT), conforme Eq.7.

$p(n-1) /(n-p) \cdot F(\alpha)_{p, n-p}=$

$=\left(2(2-1) /(2-2) \cdot F(0,05)_{2,2}=4,6\right.$

- Valor Calculado (VC), conforme Eq.8.

$$
V C=n \cdot\left(d-\widetilde{d)^{T}} \cdot\left(S_{d}\right)^{-1} \cdot\left(d_{j}-\tilde{d}\right)=3,9\right.
$$

Logo:

$$
\mathrm{VC}=3,9<V T=4,6
$$

E assim sendo, aceita-se a hipótese básica $\mathrm{H}_{0}$ a um nível de significância de 5\%, cujo resultado indica que 
não existe diferença significativa entre os valores encontrados.

$\checkmark \quad$ Resultados entre as Campanha 2 e 3

Os resultados obtidos foram:

- Valor Teórico (VT), conforme Eq.7.

$$
\begin{aligned}
& p(n-1) /(n-p) \cdot F(\alpha)_{p, n-p}= \\
& =\left(2(2-1) /(2-2) \cdot F(0,05)_{2,2}=4,6\right.
\end{aligned}
$$

- Valor Calculado (VC), conforme Eq.8.

$$
V C=n \cdot\left(d-\widetilde{d)^{T}} \cdot\left(S_{d}\right)^{-1} \cdot\left(d_{j}-\tilde{d}\right)=3,6\right.
$$

Logo:

$$
\mathrm{VC}=3,6<V T=4,6
$$

E assim sendo, aceita-se a hipótese básica $\mathrm{H}_{0}$ a um nível de significância de $5 \%$, cujo resultado indica que não existe diferença significativa entre os valores encontrados.

\section{CONCLUSÕES}

Os resultados obtidos utilizando os conceitos estatísticos serviram para demonstrar que as coordenadas das fissuras (Bloco 5, Bloco 8 e Bloco 11) avaliadas comparando duas campanhas (Campanha 1 e 4 e Campanha 2 e 3) não variam ao longo do tempo a um nível de significância de 5\%, podendo ser consideradas estáveis.

Ficou comprovado que com a automação das técnicas geodésicas para a coleta, transferência e análise dos resultados, o monitoramento geodésico de estruturas torna-se uma ferramenta importante para realizar a avaliação de comportamentos de estruturas civis.

\section{AGRADECIMENTOS}

Os autores agradecem a Agencia Nacional de Energia Elétrica (ANNEL), a Companhia Paranaense de Energia Elétrica (COPEL) pelo financiamento do projeto de pesquisa com cadastro BANPESQ: 200.301.308-8. Este trabalho é parte constituinte de dissertação de mestrado vinculado à Universidade Federal do Paraná (UFPR).

\section{REFERÊNCIAS}

GRANEMANN, D. C. Estabelecimento de uma Rede Geodésica para o Monitoramento de Estruturas: Estudo de Caso na Usina Hidrelétrica de Salto Caxias. Dissertação Apresentada ao Curso de Pós-
Graduação em Ciências Geodésicas, 112p, UFPR, Curitiba, 2005.

SILVEIRA, J. F. A. Instrumentação e Comportamento de Fundação de Barragens de Concreto. São Paulo: Oficina de Textos, 2003. 320p. 